\title{
Uncompensated Centrifugal Flow about Accelerated Cosmic Expansion
}

\author{
Luis Alberto Pérez Pérez (i) \\ Curiosidad y Exploración para el Entendimiento y el Conocimiento, Valladolid, Spain \\ Email: fenotipo01@gmail.com
}

How to cite this paper: Pérez, L.A.P. (2021) Uncompensated Centrifugal Flow about Accelerated Cosmic Expansion. World Journal of Mechanics, 11, 83-93.

https://doi.org/10.4236/wjm.2021.114007

Received: March 8, 2021

Accepted: April 25, 2021

Published: April 28, 2021

Copyright $\odot 2021$ by author(s) and Scientific Research Publishing Inc. This work is licensed under the Creative Commons Attribution International License (CC BY 4.0).

http://creativecommons.org/licenses/by/4.0/ (c) (i) Open Access

\begin{abstract}
Currently, we are under the perception of a visible universe which has an accelerated expansion, because repeated evidences obtained by several technics since the well known observations performed by Edwin Powell Hubble. The world scientific community was astonished by these observations, and since then until today, countless calculations have been made that only leave the hypothesis of the existence of an unknown cosmic entity that has the particularity of repelling matter from each other when it is sufficiently separated by huge amounts of that entity, which was called as "dark energy". This "dark energy" is a completely unknown thing, and it is understood by some researchers as the convenient hypothesis, because it is which emerges from deep calculations and observations. Bearing in mind that we already know about all cosmic objects and systems are in rotation, both locally and not so locally, and that everything is full and endowed with intrinsic and extrinsic angular momentum, it seems logical to think that rotational dynamics must also apply to the more extensive, and that if locally (and not so locally) we perceive and infer rotating objects everywhere, then they must also exist globally. So, starting from the idea that rotation is omnipresent, at every level of sizes; from the invisibly small to the invisibly large, I thought that it is really wise to cover it through rotation dynamics, or that in the worst case, we cannot ignore the fact of the omnipresent rotation in any entity to infer. And this is the main reason for the resolution and the motivation of the birth of the publication of this study. Based on this seemingly simple idea, these results and conclusions of this study was reached: following a formal logic and evidence of the accelerated unfolding of the cosmic fabric, another hypothesis is proposed as an alternative to the existence of the "dark energy": The intensities of centrifugal acceleration fluxes exceed the intensities of gravitational fluxes, which are not sufficient to compensate for the centrifugal flux, which is why baryonic and exotic matter, energy, space and time are extended, in geometric progression with respect to our apparent time. So, this unfolding of the cosmic
\end{abstract}


fabric might not be caused by a "dark energy", but by centrifugal fields of rotational-orbital domains. Besides all the above, this publication has a double purpose, because it is also intended to cover another matter; these deep attentions to the Rotational Dynamics also derive in another solution or hypothesis regarding the formed mystery after observing the incoherent too high velocity of matter in the far latitudes in spiral galaxies: The hypothesis of the existence of the so-called "dark matter" arises from the observation that, in spiral galaxies, all that matter which is located beyond a critical distance from the galactic rotation axis, travels too fast, while the calculations illustrate an insufficient intensity of gravitational acceleration flux to explain that speed and to retain all that matter while maintaining the orbital distance. However, the concept I present here, is that, in a spiral galaxy, all those matter which is positioned farthest from the main rotation axis, is effectively lost in space as time goes by, or what is the same, the intensity of the centrifugal acceleration field is not compensated by that of the gravitational field, and the destiny of every spiral galaxy is a more or less homogeneous and compact disk-shaped galaxy. As a basic NOTE to be taken into account; in the present article we intend to show the radical, meticulous and delicate relation that exists in the considerations of the classic concept of "isolated system of particles", with the degrees of the consistencies of the connections between all those "particles", whether they are inter-material bonds, or, in a relativistic scope, bonds between the same cosmic fabric. And this is so, because the idea of "isolated" falls directly and precisely on the criterion that we put to the degree of consistency of each connection between each "part" or "particle" that integrates the presumed and inferred concept of "isolated system" with respect to the rest of systems or universe.

\section{Keywords}

Fields Theory, Inertial Fields, Rotational Dynamics, Angular Momentum, Orbitation, Macroscopic Bodies Mechanics, Dark Energy, Dark Matter, Centrifugal Action, Intermolecular Bonds, Cosmic Fabric, Inter-Astral Ligatures, Gravitational Field Strength

\section{Introduction}

With the intention of inferring about what happens in the cosmic fabric, I will resort to what happens inside the material fabric of a rigid solid in rotation, as far as inertial fields are concerned.

From the result of what occurs with the rigid solid, and always with the already well known basic notions and premises of rotational dynamics, the behavior of the material particles is extrapolated to what will happen with the cosmic fabric.

The spinning top is a "rigid solid of revolution". It means that, in its intrinsic rotation about its principal axis, its intermolecular bonds are sufficient to retain 
its particles while maintaining the distance to the axis of rotation.

In other words, the intermolecular bonds compensate the centrifugal acceleration fluxes: Every molecule maintains its distance to the main axis of rotation.

To expose this, we have a clear example about what happens when, on a working lathe, the operator devastates rotating body material at the outside of the rotating piece: the centrifugal acceleration flow continues acting on the particles released from the rest of the body, which are now without its bonds.

Consequently, all those parts are detached from the rotational domain (rotating body, i.e. rotating isolated particle system), losing its angular momentum they had when they were part of the whole body, so they leave its relative positions respect the body rotational axis, causing them to go away from the body and to acquire a purely inertial rectilinear motion, distancing themselves from the global rotational axis (Figure 1).

In an equivalent manner, each cosmic fabric constitutes a specific rotational-orbital domain.

It is not detached from the domain, it is bound to it, but unlike the rigid solid of revolution, its fabric bonds are not so consistent.

This causes 2 effects:

1) The so-called "Coriolis effect", which consists of a cadence, not only of the angular velocity, but also of the angular momentum, for the parts furthest away from the main axis of rotation of the domain, and

2) The centrifugal acceleration flux intensity is greater than the gravitational flux intensity, which causes the most distants parts from the main axis of rotation-orbitation, to move away from it, exponentially respect a local timing (Figure 2).

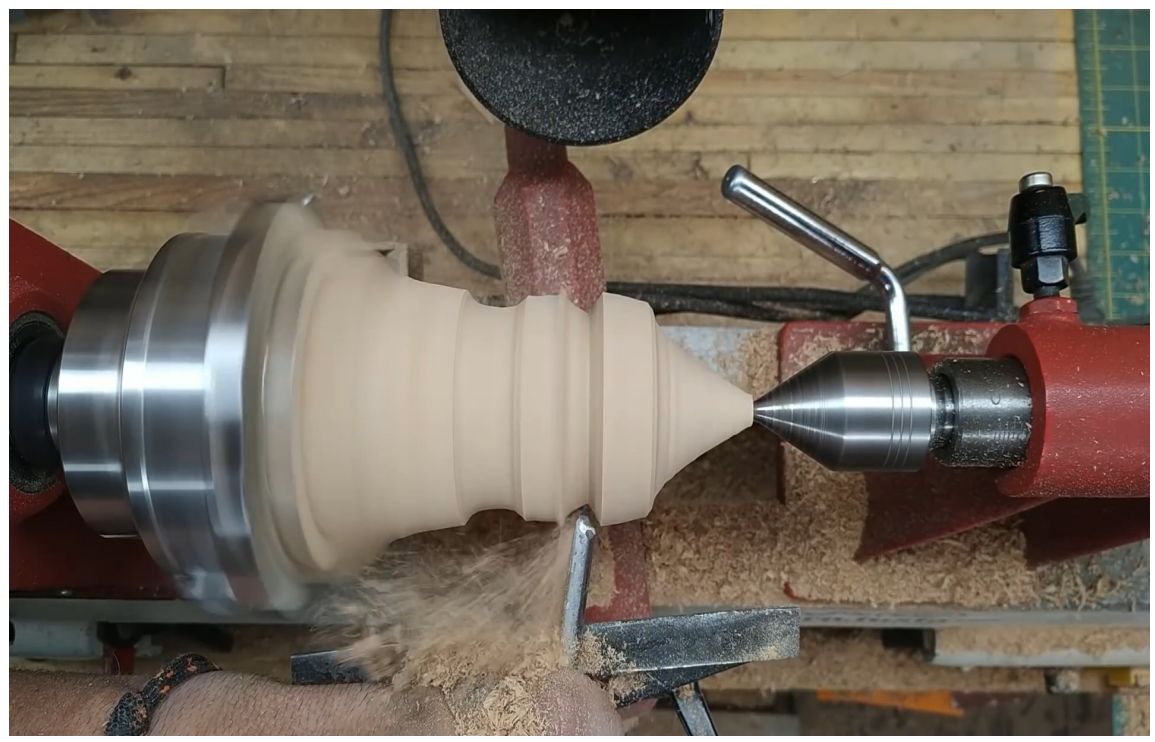

Figure 1. Lathe operator destroys some inter-molecular bonds outside the rotating body. This fact, inmediatly causes a leakage of the loose particles, which acquire an acceleration flux component, collinear to the radius of rotation and also a velocity flux component, collinear to the tangent to the radius of rotation. 


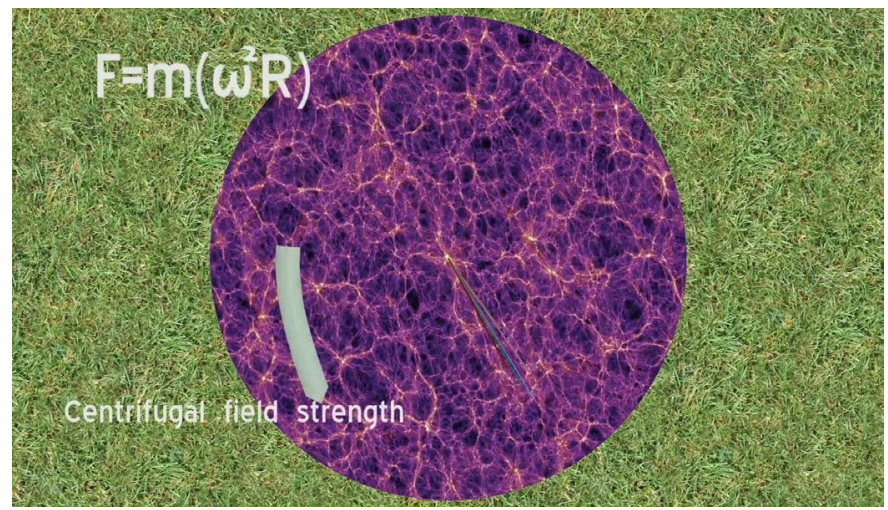

Figure 2. Any cosmic fabric, including our observable universe, could be considered as an "isolated" orbitation domain. It is suggested to watch Video 1 link for a better and clearer view of this.

\section{Main Details}

Inspired by the deliveries of Miguel Catalán Sañudo, Gabriel Barceló wrote the Theory of Dynamic Interactions, and as described in some of his publications, we find ourselves in a cosmic environment in which every considerable system as "isolated"; it rotates, and therefore, it orbits, regardless of the sizes under study.

At the instant when a non-coaxial torque is done to any rotating material entity, there appears new centrifugal interaction which makes the older one to roll into itself.

Without any simultaneous non-coaxial rotations, the centrifugal action is the main non-inertial action suffered by all the material which composes the whole system.

Work "Analysis of Inertial fields in non-inertial systems" from Barceló, is more centered about simultaneous non-coaxial torques, but the whole work treats the fact of the inertial fields which are originated by non-inertial fields, and vice versa.

The lathe operation is a great descriptive near example of that phenomena, where the materia is envolved in inertial fields from a previous status of noninertial fields [1] [2] [3].

With today's techniques and technologies, it is only possible to infer and know with certain precision the relatively "local" "isolated" rotational systems, such as the relative orientations of the axes of our planets or nearby galaxies, their angular velocities, etc., but at the dimensional scales of galactic clusters, we still cannot.

Our present knowledge allows us to know that the intrinsic rotation axis of the Earth is not parallel with that of Saturn, nor this with that of the Sun, nor this with that of the rotation of the Milky Way, nor this with that of the rotation of the Andromeda galaxy...

In other words, we know today that the stars or cosmic bodies in general are endowed with an angular momentum, with their own intrinsic rotation, which is particular to each system, that is, each star has its own rotational domain, on 
which there orbit each and every one of the parts and particles that constitute that mentioned star.

Also each astral group, or each stellar system is provided with its own angular momentum, with its angular velocity of a particular and concrete value, as well as with an orientation of its concrete and unique rotational axis.

And also the galaxy that hosts that stellar group has its own angular momentum, with its own angular velocity and its own concrete and particular orientation of its axis of rotation.

So on and so forth, so that we must realize and radically understand that: There exist as many principal axes of rotation as there are systems of particles that we consider as "isolated" in the cosmos.

It is necessary to warn here that it is a conceptual key to realize which is the criterion we use to conclude on what is, and what is not for us, a consideration of "isolated system", or to what extent it is or is not, either of particles, or, as in the case of astro-physics, of cosmic fabric.

By saying "rotational domain" we mean exactly our human consideration and criteria of; what is an isolated system of cosmic fabric, because, we have criteria on what we base to establish what is, and what is not, an "isolated system", either of particles or in the case of this publication; of cosmic fabric. And "cosmic fabric" means not only particles, but also time, space, energy or matter.

"Rotational domain" means the equivalent in the ferromagnetic matter we have at our fingertips and we can analyze today, in which ferromagnetic domains exist, which are described by the disparate internal motions of the electric charges inside the matter, as well as of the spins of the charge particles. Except in permanent magnets, matter presents a multitude of directions of the axes of rotations and spins at its particles, which are generally not parallel to each other.

In this publication, it is proposed to extrapolate this fact by arguing that the cosmos should not be treated as a globality with a global axis of rotation, but that every axis is local, referred to a concrete domain of cosmic fabric, the axes of each rotational spatial domain, depending on its locality, are not, generally, parallel to each other.

This is how the Cosmos is estimated in the Theory of Dynamic Interactions, as an astral "soup of soups", with matter and energy in multiple rotational-orbital domains; multiple non-coaxial and non-coincident axes [4]. It is considered that any system that is analyzed, is always local and relative, and that the idea of "global" is not something that we should contemplate to seriously study nature.

We do not resort to ideal inventions forced by certain observations and calculations, such as "dark matter", or "dark energy", and the explanation to this supposed spatial separation, accelerated between its parts, simply lies in the centrifugal flows in rotational-orbital domains.

These intensities of centrifugal acceleration fields exceed the intensities of gravitational fields, with gravitational bindings not being sufficient to compensate for or retain the centrifugal flows, which is why the systems distance them- 
selves from each other in geometric progression.

Establishing then that our galaxy (which has its particular angular momentum) forms a cell within a system that we call Local Group, and that this in turn is also a cell within some system that also has its own angular momentum, so we must then extend the patterns previously exposed about the rotational-orbital domains, so that we consider that we are in a rotating world that is part of another larger system that also rotates, and this one within another, and this one within another, successively, but that none of them has the same angular velocity amount, nor the same orientation of its axis, and so on.

If the known universe, or a section of it, is a mass of dust, our galaxy could be taken as a speck of dust within this large rotational-orbital domain.

In this cosmic domain, wherever our galaxy is located, our sight would perceive all other objects, or "specks of dust", moving away from us in an accelerating manner, with exponentially increasing speed in any direction in which we set our sight (Figure 3).

\section{Meaning of Centrifugal Flux}

Continuing with the notions provided by the field theory, and making conceptual use of it; in a rigid solid of revolution, as in the generality of the stars, there is an intrinsic rotation and therefore, an orbiting of each one of its particles around its center of masses.

The inter-molecular interactions that constitute this type of systems compensate the value of the field strength of normal acceleration $\left|\vec{a}_{n}\right|$ (centripetal acceleration flux strength), maintaining the same value but in the opposite direction as its centrifugal acceleration flux strength $\left|\vec{a}_{c}\right|$, holding each particle with a constant distance from the axis of rotation of the system:

$$
-\vec{a}_{n}=\vec{a}_{c}=\omega^{2} \cdot \vec{R}
$$

this is the acceleration field strength experienced by any mass or system $m$ that is part of a set or domain $M$ that is in rotation.

This mass $m$ is in orbit around an extrinsic axis, which is the rotational axis of the ensemble $M$.

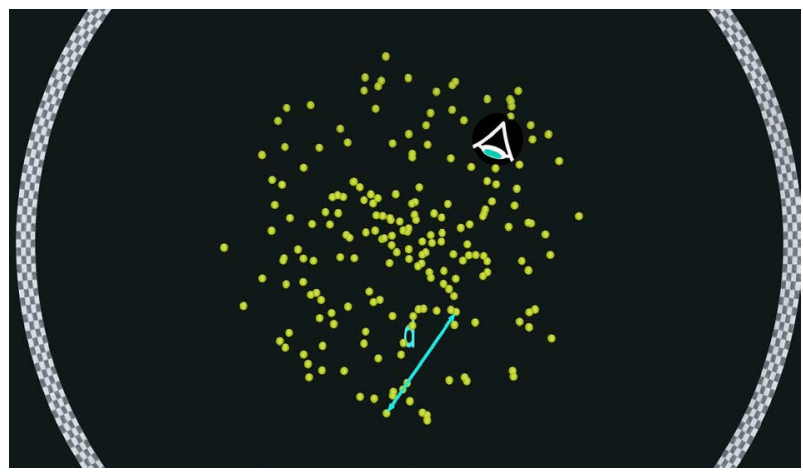

Figure 3. Didactic scheme of the position and view from our galaxy with respect to our known universe (it is suggested to watch Video 1 link for a better and clearer view of this). 
As this equation shows, the acceleration field strength $\vec{a}_{c}$ experienced by any orbiting mass, grows as a straight-line function of the constant $\omega^{2}$ (square of the angular velocity) of the rotation-orbitation, and also as a straight-line function of the distance to the rotational axis around which the system orbits $(\vec{R})$.

So well, the obtained result from this, is that when an orbiting system $m$ is not provided with some rigid fixation to the rest of the set or rotational domain $M$ to which it belongs, that is, if it is not strongly united, with a sufficient consistency with the other systems or parts that orbit $\left(m_{1}, m_{2}, m_{3}, \cdots, m_{n}\right)$, then the orbiting matter $m$ ceases to maintain its distance $(|\vec{R}|)$ to its orbiting axis, and moreover, this distance increases as a function of a time referred to any observer, whether this observer is located within the rotational set or domain $M$ itself, or whether it is an observer completely outside the rotating set: both observers will notice that the system $m$ acquires an increase of its velocity with respect to the rotational center.

Unlike a rigid solid, the known universe lacks sufficiently rigid bonds between its parts. The normal accelerating field strength succumbs to the centrifugal one:

$$
\left|\vec{a}_{n}\right|<\left|\vec{a}_{c}\right| ;\left|\vec{a}_{n}\right|<\left|\omega^{2} \cdot \vec{R}\right|
$$

The logical result of this would be that the non-rigidly bound parts or systems orbit away exponentially from their own extrinsic axes of rotation (orbital axes):

Each galaxy in the universe distances its own distance to an extrinsic axis of rotation around which it orbits, which is common not only to galaxy clusters but to rotational-orbital domains of the cosmos.

Being $\vec{R}$ the distance from the center of a particular galaxy to its extrinsic orbital axis, this $\vec{R}$ grows in time in a rectilinear fashion, its growth rate being the constant $\omega^{2}$, which is the same for each and every part included in the same concrete orbital domain, that we consider it as an isolated (domain) rotational-orbital system.

However, this implies something different. Looking at the above equation, we see that as any local time elapses, the value of the acceleration field strength $\vec{a}_{c}$ is made up according to the value of the distance $\vec{R}$ at that theoretical instant, so that this distance $\vec{R}$ is increased according to an exponential graph or function, and this would also implies that each position of each particle in the cosmic fabric within that domain, distances itself from any other, and not in a rectilinear way, but exponentially, accelerating in our local time of observation.

To conceive this fact mentally without resorting to the didactic video attached and referred to below in this article, let us imagine a merry-go-round in which there are horses and other elements and objects; each object is separated by a different distance from the center of rotation $O$ at the merry-go-round.

It so happens, then, that the tendency to move away from the center $O$ of rotation, is greater for the horses and objects more distant from $O$, than for the ones closer to $O$.

Those more distant objects are accelerated positions with respect to $O$, with greater intensity than those closer to. 


\section{Comparison to Gravity}

And it is interesting to make here a brief parenthesis on the comparison between the nature of the gravitational field strength $\vec{g}$ and the nature of the centrifugal field strength $\vec{a}_{c}$ :

About the first, since Galileo it was already discovered, through his inclined planes, that the distance $d$ traveled by any mass in free fall on the earth's crust, is proportional to the square of the times: $d=$ const $\cdot t^{2}=\frac{1}{2} g \cdot t^{2}$, where $t$ would be units of local time.

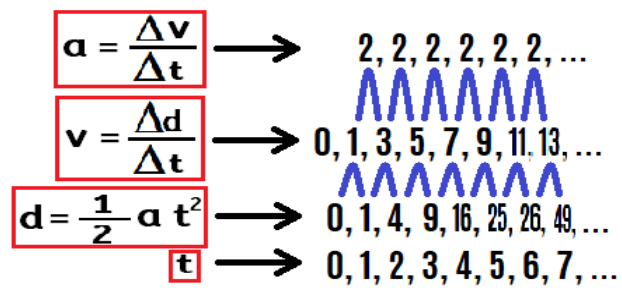

This is on the assumption of $a$ with a constant value (" 2 " in this case), however we know that when we treat the gravity, this value undergoes an increase as a function of distance $d$, namely its square $d^{2}$.

However, the nature of the gravitational flux intensity $g$ is such that, in relation to the instantaneous distance of an object from a center of gravity, it presents a curve not only with the square of the local time $t^{2}$, but also with the square of the distance $d^{R}$, since $\frac{1}{2} a$ does not remain constant, as Galileo presented it, but it increases as the object approaches the center of gravity, and its rate of increase corresponds to the square of that distance $\left(d^{2}\right)$.

Thus, the acceleration concluded by Galileo turns out to be only at the local level; that is, when $\frac{1}{2} a$ is constant in time and in the position of the object.

However, in nature this value is not constant; the distance of the object being increased by a gradient of power " 4 ".

On the other hand we have another type of accelerating inertial field:

The centrifugal field strength $\vec{a}_{c}$.

This centrifugal field strength $\vec{a}_{c}$ presents another nature, and therefore, another different gradient than $\vec{g}$.

Specifically, this gradient presents an increment of power " 3 " from its center of orbit with respect any local time, because the incremental rate of its acceleration, instead of being the square of the distance (as it happens with gravity $\vec{g}$ ), it is of the simple distance, as reflected in the equation: $\vec{a}_{c}=\omega^{2} \cdot \vec{R}$, where $\vec{R}$ is that distance or position of the center of masses of an object from its orbitation axis (Figure 4).

In order to have a numerical view about the explained gradient differences, here is a list with a numeric sequence of a gravitational field free fall gradient compared to a centrifugal field free fall gradient. 
It is a sequence in which the position (distance), velocity and acceleration of center of masses of any free falling body, are recorded at identical local time intervals. These values are relative to the position of a point away from the center of gravity (gravitational case), and relative to the axis of rotation (centrifugal case):

\begin{tabular}{|c|c|c|}
\hline \multicolumn{3}{|c|}{ Gravitational field free fall gradient } \\
\hline Pos. & Vel. & Acc. \\
\hline 7E6 & 0 & 0 \\
\hline $7 \mathrm{E} 6$ & $-2.83 \mathrm{E} 2$ & $-2.83 \mathrm{E} 2$ \\
\hline $7 \mathrm{E} 6$ & $-1.13 \mathrm{E} 3$ & $-8.5 \mathrm{E} 2$ \\
\hline 7E6 & $-2.55 \mathrm{E} 3$ & $-1.42 \mathrm{E} 3$ \\
\hline $7 \mathrm{E} 6$ & $-4.5 \mathrm{E} 3$ & $-1.99 \mathrm{E} 3$ \\
\hline $7 \mathrm{E} 6$ & $-7.1 \mathrm{E} 3$ & $-2.56 \mathrm{E} 3$ \\
\hline 7E6 & $-1.02 \mathrm{E} 4$ & $-3.14 \mathrm{E} 3$ \\
\hline 7E6 & $-1.4 \mathrm{E} 4$ & $-3.7 \mathrm{E} 3$ \\
\hline $6.9 \mathrm{E} 6$ & $-1.83 \mathrm{E} 4$ & $-4.4 \mathrm{E} 3$ \\
\hline $6.9 \mathrm{E} 6$ & $-2.33 \mathrm{E} 4$ & $-5 \mathrm{E} 3$ \\
\hline $6.9 \mathrm{E} 6$ & $-2.9 \mathrm{E} 4$ & $-5.7 \mathrm{E} 3$ \\
\hline $6.9 \mathrm{E} 6$ & $-3.5 \mathrm{E} 4$ & $-6.4 \mathrm{E} 3$ \\
\hline $6.8 \mathrm{E} 6$ & $-4.3 \mathrm{E} 4$ & $-7.2 \mathrm{E} 3$ \\
\hline $6.8 \mathrm{E} 6$ & $-5.1 \mathrm{E} 4$ & $-8 \mathrm{E} 3$ \\
\hline $6.7 \mathrm{E} 6$ & $-6 \mathrm{E} 4$ & $-9 \mathrm{E} 3$ \\
\hline $6.6 \mathrm{E} 6$ & $-7 \mathrm{E} 4$ & $-1 \mathrm{E} 4$ \\
\hline $6.6 \mathrm{E} 6$ & $-8.1 \mathrm{E} 4$ & $-1.12 \mathrm{E} 4$ \\
\hline $6.5 \mathrm{E} 6$ & $-9.3 \mathrm{E} 4$ & $-1.27 \mathrm{E} 4$ \\
\hline $6.3 \mathrm{E} 6$ & $-1.08 \mathrm{E} 5$ & $-1.44 \mathrm{E} 4$ \\
\hline $6.2 \mathrm{E} 6$ & $-1.24 \mathrm{E} 5$ & $-1.64 \mathrm{E} 4$ \\
\hline $6.1 \mathrm{E} 6$ & $-1.43 \mathrm{E} 5$ & $-1.9 \mathrm{E} 4$ \\
\hline $5.9 \mathrm{E} 6$ & $-1.65 \mathrm{E} 5$ & $-2.22 \mathrm{E} 4$ \\
\hline $5.7 \mathrm{E} 6$ & $-1.92 \mathrm{E} 5$ & $-2.64 \mathrm{E} 4$ \\
\hline $5.5 \mathrm{E} 6$ & $-2.24 \mathrm{E} 5$ & $-3.2 \mathrm{E} 4$ \\
\hline $5.2 \mathrm{E} 6$ & $-2.64 \mathrm{E} 5$ & $-4 \mathrm{E} 4$ \\
\hline $4.9 \mathrm{E} 6$ & $-3.2 \mathrm{E} 5$ & $-5.2 \mathrm{E} 4$ \\
\hline $4.5 \mathrm{E} 6$ & $-3.9 \mathrm{E} 5$ & $-7.1 \mathrm{E} 4$ \\
\hline $4 \mathrm{E} 6$ & $-4.9 \mathrm{E} 5$ & $-1.05 \mathrm{E} 5$ \\
\hline $3.4 \mathrm{E} 6$ & $-6.7 \mathrm{E} 5$ & $-1.74 \mathrm{E} 5$ \\
\hline $2.35 \mathrm{E} 6$ & $-1.02 \mathrm{E} 6$ & $-3.6 \mathrm{E} 5$ \\
\hline $8.8 \mathrm{E} 4$ & $-2.26 \mathrm{E} 6$ & $-1.24 \mathrm{E} 6$ \\
\hline
\end{tabular}


Centrifugal field free fall gradient

\begin{tabular}{|c|c|c|}
\hline Pos. & Vel. & Acc. \\
\hline $1 \mathrm{E}-1$ & 0 & 0 \\
\hline $1.01 \mathrm{E}-1$ & $5 \mathrm{E}-4$ & $5 \mathrm{E}-4$ \\
\hline $1.03 \mathrm{E}-1$ & $2.01 \mathrm{E}-3$ & $1.51 \mathrm{E}-3$ \\
\hline $1.07 \mathrm{E}-1$ & $4.6 \mathrm{E}-3$ & $2.6 \mathrm{E}-3$ \\
\hline $1.16 \mathrm{E}-1$ & $8.6 \mathrm{E}-3$ & $4 \mathrm{E}-3$ \\
\hline $1.3 \mathrm{E}-1$ & $1.45 \mathrm{E}-2$ & $5.9 \mathrm{E}-3$ \\
\hline $1.54 \mathrm{E}-1$ & $2.34 \mathrm{E}-2$ & $9 \mathrm{E}-3$ \\
\hline $1.91 \mathrm{E}-1$ & $3.8 \mathrm{E}-2$ & $1.42 \mathrm{E}-2$ \\
\hline $2.52 \mathrm{E}-1$ & $6.1 \mathrm{E}-2$ & $2.36 \mathrm{E}-2$ \\
\hline 0.35 & $1.02 \mathrm{E}-1$ & $4.1 \mathrm{E}-2$ \\
\hline 0.53 & $1.77 \mathrm{E}-1$ & $7.5 \mathrm{E}-2$ \\
\hline 0.85 & 0.32 & $1.45 \mathrm{E}-1$ \\
\hline 1.47 & 0.61 & $2.93 \mathrm{E}-1$ \\
\hline 2.71 & 1.24 & 0.63 \\
\hline 5.4 & 2.66 & 1.41 \\
\hline $1.14 \mathrm{E} 1$ & 6 & 3.4 \\
\hline $2.6 \mathrm{E} 1$ & $1.46 \mathrm{E} 1$ & 8.6 \\
\hline $6.4 \mathrm{E} 1$ & $3.8 \mathrm{E} 1$ & $2.3 \mathrm{E} 1$ \\
\hline $1.66 \mathrm{E} 2$ & $1.03 \mathrm{E} 2$ & $6.5 \mathrm{E} 1$ \\
\hline 4.7E2 & $3.01 \mathrm{E} 2$ & $1.98 \mathrm{E} 2$ \\
\hline $1.4 \mathrm{E} 3$ & $9.3 \mathrm{E} 2$ & $6.3 \mathrm{E} 2$ \\
\hline $4.5 \mathrm{E} 3$ & $3.09 \mathrm{E} 3$ & $2.16 \mathrm{E} 3$ \\
\hline $1.54 \mathrm{E} 4$ & $1.09 \mathrm{E} 4$ & $7.8 \mathrm{E} 3$ \\
\hline $5.6 \mathrm{E} 4$ & $4.1 \mathrm{E} 4$ & $2.98 \mathrm{E} 4$ \\
\hline $2.17 \mathrm{E} 5$ & $1.61 \mathrm{E} 5$ & $1.21 \mathrm{E} 5$ \\
\hline 9E5 & $6.8 \mathrm{E} 5$ & $5.2 \mathrm{E} 5$ \\
\hline $3.9 \mathrm{E} 6$ & $3.03 \mathrm{E} 6$ & $2.35 \mathrm{E} 6$ \\
\hline $1.82 \mathrm{E} 7$ & $1.43 \mathrm{E} 7$ & $1.13 \mathrm{E} 7$ \\
\hline 9E7 & 7.1E7 & $5.7 \mathrm{E} 7$ \\
\hline $4.7 \mathrm{E} 8$ & $3.8 \mathrm{E} 8$ & $3.06 \mathrm{E} 8$ \\
\hline $2.57 \mathrm{E} 9$ & $2.1 \mathrm{E} 9$ & $1.72 \mathrm{E} 9$ \\
\hline
\end{tabular}




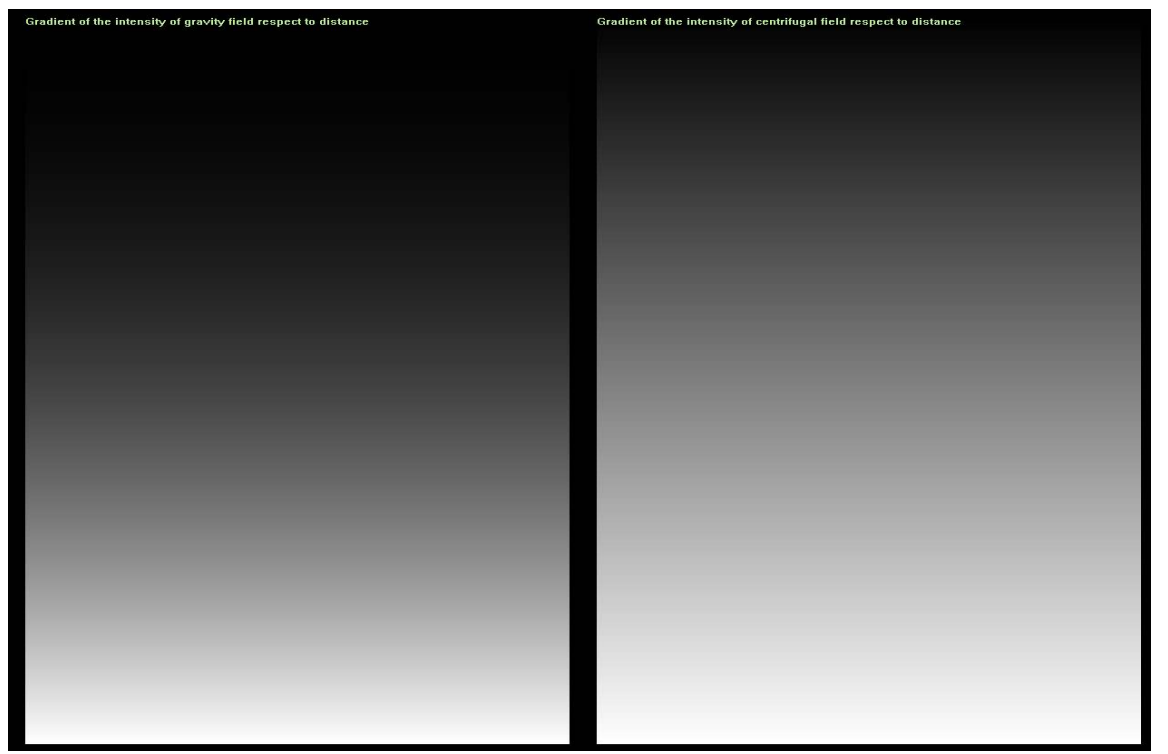

Figure 4. Gradients of the gravitational field strength $\vec{g}$ and of the centrifugal field strength $\vec{a}_{c}$ with respect to the position or distance of an object from a center of gravity, and with respect to the distance from a center of rotation, respectively.

\section{Conclusions}

The above is therefore, a logical alternative to the proposal of the existence of "dark energy", because the exponential gap could naturally be caused by a decompensation between the centripetal acceleration field strength and the centrifugal acceleration field strength:

$$
\left|\vec{a}_{n}\right|<\left|\vec{a}_{c}\right| ;\left|\vec{a}_{n}\right|<\left|\omega^{2} \cdot \vec{R}\right|
$$

In short, the present conjecture proposes an overwhelmingly simple explanation for the accelerated separation between galaxies and galaxy clusters, and could explain at least part of the observations and experiments dated to date.

There is also a video annex for a didactic presentation of this publication, which can be watched: https://www.youtube.com/watch?v=tCPM3PMp6Tc Video 1 link.

\section{Conflicts of Interest}

The author declares no conflicts of interest regarding the publication of this paper.

\section{References}

[1] Barceló, G. (2012) Analysis of Dynamics Fields in Noninertial Systems. WJM, 2, 175-180.

[2] Barceló, G. (2008) Un mundo en rotación. Marcombo, Barcelona.

[3] Barceló, G. (2013) Imago Universi: A Story of the Human Conception of the Cosmos. Arpegio, Barcelona. http://www.editorialarpegio.com/ http://advanceddynamics.net/imago-universi/

[4] Barceló, G. (2017) New Paradigm in Physics, Vols 1 to 4: Theory of Dynamic Interactions. Amazon. 